\title{
Bericht über die im hygienischen Laboratorium der Universität Berlin ausgeführten Untersuchungen des Berliner Leitungs- wassers in der Zeit vom 1. Juni 1885 bis 1. April 1886. $\left.{ }^{1}\right)$ \\ Von
}

\author{
Prof. Dr. R. Koch, \\ Geheimer Medizinalrat, Direktor der hrgienischen Institute.
}

Für die Entnahme der Wasserproben wurde dasselbe Programm eingehalten, welches bei Übernahme der Untersuchung seitens des Kaiserlichen Gesundheitsamtes durch das Mitglied dieses Amtes Herrn Regierungsrat Dr. Wolf $\mathrm{f}$ hügel mit dem Direktor der städtischen Wasserwerke Herm Gill vereinbart worden ist. Diesem Übereinkommen zufolge wurde die Entnahme der zu untersuchenden Wasserproben durch Beamte der städtischen Wasserwerke nach einer hierfür im Juni 1885 im Laboratorium des Kaiserlichen Gesundheitsamtes erhaltenen Anleitung ausgeführt und hat bis jetzt ohne Unterbrechung stattgefunden.

Alle acht Tage, und zwar am Dienstag, erfolgte der oben erwähnten Vereinbarung gemäß die Entnahme von 10 Wasserproben an folgenden Stellen:

1. Am Stralauer Werk an der Schöpfstelle,

2. Am Stralauer Werk nach der Filtration im Reinwasserbehälter,

3. Am Tegeler Werk an der Schöpfstelle,

4. Am Tegeler Werk nach der Filtration im Reinwasserbehälter,

5. Im Charlottenburger Sammelbehälter,

6. W. Wilhelmstraße Nr. 75 (Küche des Kastellans im Auswärtigen Amt),

7. SW. Friedrichstraße Nr. 41/42 (Friedrich-Wilhelms-Gymnasium),

8. SO. Schmidtstraße Nr. 16 (Gemeindeschule),

9. N. Friedrichstraße 126 (Friedrichs-Gymnasium),

10. C. Weinmeisterstraße Nr. 15 (Sophien-Gymnasium).

Die Untersuch ung des Wassers erstreckte sich:

1. auf eine Vorprüfung, bei welcher die Klarheit, Farbe, der Geschmack und Geruch berücksichtigt wurden. Die Temperaturen der Wasserproben wurden von den die Entnahme vollziehenden Beamten unmittelbar nach der Entnahme festgestellt.

2. Auf die chemische Zusammensetzung, wobei quantitativ stets der Rückstand, der Glühverlust, das Chlor, der Kalk und die zur Oxydation der im Wasser befindlichen oxydierbaren Stoffe erforderliche Chamaeleon-Menge (Oxydierbarkeit) ermittelt worden sind. Wo die qualitative Analyse des Wassers, welche

1) Verlag von Julius Springer, Berlin, 1887. 
sich außer auf Chloride und Kalk noch auf Sulfate, Eisen, Schwefelwasserstoff, Nitrate, Nitrite und Ammoniak erstreckte, es für angezeigt erscheinen ließ, sollte auch eine quantitative Bestimmung dieser letzteren Bestandteile erfolgen. Es sei gleich an dieser Stelle erwähnt, daß außer Bestimmungen des Ammoniaks diejenigen der anderen genannten Verbindung in keinem Falle notwendig wurden.

3. Auf die bakteriologische Untersuchung des Wassers.

Die zur Ermittelung der ad 1 und 2 genannten Eigenschaften bzw. Bestandteile des Wassers angewandten Methoden waren die gleichen, welche im Laboratorium des Kaiserlichen Gesundheitsamtes bei der Untersuchung des städtischen Leitungswassers benutzt und dem Magistrat s. Z . berichtet worden sind.

Was die B esch affenheit zunächst des unfiltrierten Spree- und Seewassers anbetrifft, wie sich dieselbe den Ergebnissen der chemischen Analyse vom 1. Juni 1885 bis 1. April 1886 zufolge darstellt, so kann man sagen, daß jene im großen und ganzen die gleiche, wie in der Versuchszeit vom 1. Juli 1884 bis 1. April 1885, geblieben ist.

Die Schwankungen hinsichtlich der chemischen Zusammensetzung sowohl des Spree- als auch des Seewassers in unfiltriertem Zustande waren verhältnismäßig sehr geringe. Nur ein einziges Mal (am 30. März 1886) wurde bei dem Wasser aus dem Tegeler See eine bedeutende Verminderung der bei der quantitativen Untersuchung gewöhnlich bestimmten Bestandteile wahrgenommen. An diesem Tage war zurückgegangen

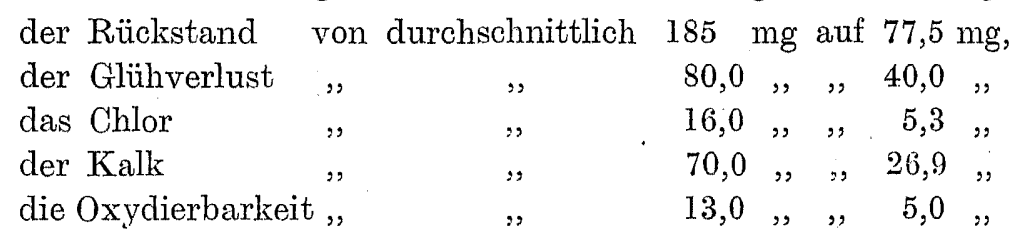

Das filtrierte Seewasser hat an dem nämlichen Tage keine bemerkenswerte Änderung gezeigt.

Eine ähnliche Erscheinung bot das nämliche Wasser am 10. und 24. Februar 1885 dar, wie aus dem Berichte des Kaiserlichen Gesundheitsamtes ersichtlich ist; dieselbe konnte seitens dieser Behörde nicht mit genügender Sicherheit aufgeklärt werden. Wie neuere Nachforschungen dartaten, hängt diese abweichende Beschaffenheit des Wassers mit dem starken Eisgang auf dem Tegeler See zusammen. Das Eis schmolz an diesem Tage stark und es war also vermutlich viel Schmelzwasser (Eiswasser) in der geschöpften Probe.

Ähnliche Verhältnisse haben, nach einer mündlichen Mitteilung des BetriebsIngenieur Herrn Ankla m m, zu Tegel am 10. und 24. Februar 1885 obgewaltet. Bei einem Vergleich der chemischen Zusammensetzung des unfiltrierten Spreewassers mit dem unfiltrierten Seewasser findet man schon früher gemachte Beobachtungen wieder bestätigt, daß das Flußwasser reicher an Chloriden und an oxydierbaren Substanzen ist als das Tegeler Wasser. Besonders der Unterschied im Chlorgehalte tritt so typisch hervor, daß man aus demselben, da er durch die Filtration nicht geändert wird, bei den in der Stadt entnommenen Wasserproben sofort erkennen kann, ob filtriertes Spree- oder Seewasser vorliegt. Für die Rückstandsmenge, den Glühverlust und Kalk kann übereinstimmend mit den im Kaiserlichen Gesundheitsamt gemachten Beobachtungen ein solch typisches Verhalten der einzelnen Wasserbezugsquellen nicht gefunden werden.

Besonders hervorgehoben sei noch, daß das Spreewasser an manchen Entnahmetagen einen ganz bedeutenden Gehalt an Ammoniak enthielt, so z. B. am 13. Oktober 1885 2,5 mg, am 9. März 1886 1,25 mg, am 23. März sogar 12,5 mg und am 30. desselben Monats $10 \mathrm{mg}$, während im Oktober 1884 der höchste Gehalt an Ammoniak 0,1 mg, im Februar $18850,16 \mathrm{mg}$ betrug und im März 1885 nur Spuren aufzufinden waren. Das unfiltrierte 
Tegeler Seewasser enthielt meistens nur Spuren und höchst selten bestimmbare Mengen von Ammoniak; der höchste, nur einmal beobachtete Ammoniakgehalt betrug $0,6 \mathrm{mg}$ (am 9. März 1886). Die Ursache für die oben angeführten, a uffallend hohen Ammoniakmengen im unfiltrierten Spreewasser konnten nicht eruiert werden.

Weder im unfiltrierten Spreewasser noch im unfiltrierten Seewasser waren Nitrite, Nitrate (angewandt wurde die Metaphenylendiamin-Reaktion in Verbindung mit der Jodzink-Stärke-Reaktion und die Diphenylamin-Probe), Sulfide und Schwefelwasserstoff nachweisbar; die Eisenmengen, sowie der Gehalt an Sulfaten war, nach dem Ausfall der qualitativen Reaktionen zu urteilen, ein sehr geringer.

Bezüglich der äußeren Eigenschaften ist folgendes zu resümieren: Die Farbe des unfiltrierten Spreewassers war gelblich, die des Wassers aus dem Tegeler See bald gelblich, bald nur schwach gelblich; beide Wässer waren trübe, enthielten bald mehr, bald weniger suspendierte Bestandteile, rochen und schmeckten mehr oder weniger modrig und setzten beim Stehen bald stärkere, bald schwächere Bodensätze $a b$.

Wie die chemische Untersuchung der Wässer n a c h i h re r Filtrat i o n erwies, übte letztere in erster Linie und hauptsächlich auf die äußeren Eigenschaften des Wassers einen günstigen Einfluß aus. Die filtrierten Wässer - sowohl die aus den Reinwasserbehältern der Stralauer und Tegeler Werke und aus dem Charlottenburger Hochbehälter, als auch die in der Stadt an den bezeichneten 5 Stellen entnommenen Proben - erwiesen sich als klar, schwach gelblich, ohne modrigen Geruch und Geschmack und nur in vereinzelten Fällen waren geringe Bodensätze zu konstatieren.

In der chemischen Beschaffenheit wurde durch die Filtration im Gehalt, an Rückstand, Chloriden und Kalk gegenüber den nicht filtrierten Wässern nur wenig geändert. Dagegen fand nach der Filtration regelmäBig eine Verminderung des Glühverlustes, der Oxydierbarkeit und im Ammoniakgehalt statt; in bezug auf den letzteren trat dieselbe selbst dann hervor, wenn das unfiltrierte Wasser auffallend hohe Mengen von Ammoniak aufwies.

So z. B. war das filtrierte Spreewasser am 13. Oktober 1885 frei von Ammoniak, während das unfiltrierte Spreewasser an demselben Tage 2,5 mg Ammoniak enthielt; am 9. März 1886, an dem dasselbe Wasser 1,25 mg, am 23. März und 30. März, an welchen Tagen 12,5 bzw. $10 \mathrm{mg}$ Ammoniak im Liter nachzuweisen waren, wurden in den filtrierten Wasserproben nur Spuren davon aufgefunden. Nur am 2. Februar enthielt das filtrierte, aus dem Reinwasserbehälter entnommene Spreewasser ebensoviel Ammoniak, als das unfiltrierte, nämlich $0,8 \mathrm{mg}$ im Liter, dagegen waren an diesem Tage alle übrigen filtrierten Wässer, - selbst die anscheinend fast nur vom Stralauer Werke kommenden Wasser vom Grundstücke Schmidtstraße Nr. 16 und Weinmeisterstraße Nr. 15 - nur mit Spuren von Ammoniak behaftet.

Das Wasser des Charlottenburger Sammelbehälters, welcher von den Tegeler Werken versorgt wird, zeigte mitunter in seinen äußeren Eigenschaften im Vergleich mit dem filtrierten Tegeler Wasser Unterschiede, welche darauf hindeuten, daß das Wasser entweder bei seinem Laufe von Tegel nach Charlottenburg, oder aber im Sammelbehälter selbst Verunreinigungen aufgenommen hatte. Das dem Charlottenburger Sammelbehälter entnommene Wasser war nämlich in einigen Fällen trübe, enthielt häufig suspendierte Bestandteile meist vegetabilischen Ursprungs, häufig auch mit bloßem Auge sichtbare kleine Süßwasserbewohner (Daphnia, Pulex, Anguilula, Wasserpolypen u. dgl. m.); die aus dem Reinwasserbehälter der Tegeler Werke zur Untersuchung eingesandten Proben dagegen waren stets frei davon. Die Ursache dieser ungünstigen Veränderung des filtrierten Tegeler Wassers, welches aus dem Charlottenburger Hochbehälter entnommen worden ist, wird zurzeit durch Untersuchungen von Wasserproben, welche aus dem 
Sammelbehälter sowohl, als auch aus den Saugekammern stammen, zu ergründen versucht. Im Rohrnetz hat das Wasser die erwähnte ungünstige Beschaffenheit wieder verloren, denn die in der Stadt entnommenen fünf Wasserproben erwiesen sich stets frei von den im Wasser des Charlottenburger Sammelbehälters beobachteten Verunreinigungen.

Über die T e m p r a tur des Wassers und deren Veränderung in der Rohrleitung gibt nachstehende Tabelle Aufschluß, in welcher die beobachteten TemperaturMaxima und -Minima verzeichnet sind:

\begin{tabular}{|c|c|c|c|c|c|c|c|c|c|c|}
\hline & $\begin{array}{l}\text { Juni } \\
{ }^{\circ} \mathrm{C} \\
\end{array}$ & $\begin{array}{l}\text { Juli } \\
0 \mathrm{C} \\
\end{array}$ & $\begin{array}{c}\text { Angust } \\
0 \mathrm{C} \\
\end{array}$ & $\begin{array}{c}1885 \\
\text { September } \\
0 \mathrm{C}\end{array}$ & $\begin{array}{c}\text { Oktober } \\
0 \mathrm{C}\end{array}$ & $\begin{array}{c}\text { Novbr. } \\
0 \mathrm{C}\end{array}$ & $\left|\begin{array}{c}\text { Dezbr. } \\
0 \mathrm{C}\end{array}\right|$ & $\mid \begin{array}{c}\text { Januar } \\
0 \mathrm{C}\end{array}$ & $\begin{array}{c}1886 \\
\begin{array}{c}\text { Februar } \\
0 \mathrm{C}\end{array}\end{array}$ & $\begin{array}{c}\text { Mäız } \\
{ }^{0} \mathrm{C} \\
\end{array}$ \\
\hline . & 13 & 718,5 & -19 & 12 & $8-$ & $-5,5$ & -1 & 1.5 & 0.5 & $0,5-2$ \\
\hline $\begin{array}{c}\text { Spreewasser } \\
\text { filtriert . }\end{array}$ & $13,6-24$ & $18-23,6$ & $14,5-18,5$ & $11,2-16$ & $9,2-14,5$ & $3-6,7$ & 1,5 & $\begin{array}{ll}1 & -2\end{array}$ & 1 & $0,5-3$ \\
\hline $\begin{array}{l}\text { Seewasser } \\
\text { unfiltriert. }\end{array}$ & $\mid 15,1-23,4$ & $19,7-24,2$ & $16-19,4$ & $14,3-16,2$ & $9,4-12,8$ & $4,2-7,9$ & $1,5-3,6$ & $0,7-2,5$ & $0,6-0,9$ & $0,5-4,9$ \\
\hline $\begin{array}{l}\text { Seewasser } \\
\text { filtriert .. }\end{array}$ & $16,5-22,3$ & $20,8-24,2$ & $16,5-19,9$ & $14,8-16,5$ & $10,6-12,4$ & $4,7-8$ & $2,2-4,7$ & & $2,6-3$ & $3-6,5$ \\
\hline $\begin{array}{l}\text { Charlotten- } \\
\text { burg. Sam- } \\
\text { melbeh. . }\end{array}$ & $17,3-24,6$ & $21,3-24,6$ & $16,7-20$ & $15,1-16,6$ & $10,5-18,8$ & $4,8-8,1$ & $2,3-4$ & $\left|\begin{array}{ll}2 & -2,2\end{array}\right|$ & $2,2-2,3 \mid$ & $2,2-2,7$ \\
\hline $\begin{array}{l}\text { Wilhelmstr. } \\
\mathrm{Nr} .75 . .\end{array}$ & $15,5-20,5$ & $19,4-21,5$ & $16,7-19,6$ & $15-16,5$ & $11,2-13,9$ & $6,2-9,7$ & $4-5,7$ & $\mid 3,4-3,8$ & $|3-3,4|$ & $2,8-3,1$ \\
\hline $\begin{array}{c}\text { Friedrichstr } \\
\text { Nr. } 41 / 42 .\end{array}$ & $14,4-20,6$ & $18,8-20,8$ & $16,5-19,5$ & $14,8-16,7$ & $11,1-13,8$ & $6,4-9,5$ & 4 & & $3,1-4,5$ & $2,2-3,3$ \\
\hline $\begin{array}{l}\text { Schmidtstr. } \\
\text { Nr. 16... }\end{array}$ & $15,1-20,2$ & $18,6-20,9$ & $16,1-19$ & $15-16,7$ & $10,8-14$ & $5,8-9,5$ & $4,2-5,8$ & & & $3,0-3,1$ \\
\hline $\begin{array}{l}\text { Friedrichstr. } \\
\text { Nr. } 126 .\end{array}$ & $16-19,5$ & $18,7-20,7$ & $16,8-19,8$ & $15-16,7$ & $11,6-14$ & $6,3-9,8$ & $5,9-7,4$ & $4,1-5,5$ & 4 & $2,7-4,9$ \\
\hline $\begin{array}{l}\text { Weinmei- } \\
\text { sterstr. } \\
\text { Nr. } 15 . .\end{array}$ & $14,7-23,0$ & $18,2-21,5$ & $13,6-19,5$ & $14,2-17$ & $10,2-14,2$ & $6,5-9,1$ & $5,3-$ & $4,3-6$ & $3,3-5$ & $4,3-4,9$ \\
\hline
\end{tabular}

Die bakteriologis che Untersuchung wurde ebenso, wie früher im Kaiserlichen Gesundheitsamt, sowohl mit dem Mikroskop, als auch mit Hilfe der Reinkultur auf festem Nährboden ausgeführt. Um Untersuchungsfehler auszuschließen, kamen jedesmal 2 Proben, gewöhnlich mit 1 und $1 / 2 \mathrm{ccm}$ Wasser, zur Untersuchung. Die Übereinstimmung der Ergebnisse bewies dann die Richtigkeit des Versuchs.

Die $\mathrm{E} r \mathrm{~g} \mathrm{e} \mathrm{b} \mathrm{n}$ is s e der bakteriologischen Untersuchung sind in der nachstehenden Tabelle in der Weise übersichtlich zusammengestellt, daß die horizontalen Spalten den verschiedenen Jahreswochen, die vertikalen Spalten den einzelnen Entnahmestellen entsprechen. Die Zahlen geben die Menge der gefundenen Mikroorganismen, auf $1 \mathrm{ccm}$ berechnet, an. Ein Blick auf die Tabelle zeigt, daß während des Beobachtungszeitraums die Leistungen sowohl der Stralauer als auch der Tegeler Wasserwerke und ebenso die Beschaffenheit des Leitungswassers in der Stadt durchweg den Anforderungen entsprochen haben.

Die vorgekommenen und folglich genauer zu besprechenden Unregelmäßigkeiten traten dabei ohne Weiteres deutlich hervor.

1. Beschaffenheit des Wassers vor der Filtration; Vergleich des S.preewas sers mit dem Wasser des Tegeler Sees.

Das S p reew a s s e r erwies sich konstant und sehr erheblich stärker mit organischen Keimen verunreinigt als das Wasser des Tegeler Sees. Dabei waren die Schwan- 
kungen in seinem Keimgehalt sehr beträchtliche, von 960 (am 8. September) bis 110000 und 100000 (am 21. Juli und 30. März). Auch innerhalb eines und desselben Monats waren Schwankungen um das 5-10fache nichts Seltenes. Eine bestimmte Beziehung zur Temperatur und zur. Jahreszeit ließ sich nicht erkennen, da beispielsweise die Zahlen des Monats Juni von denen des Dezember, diejenigen des September von denen des Februar nicht wesentlich verschieden sind. Größeren Einfluß dürften Differenzen im Wasserstande und in der Stromgeschwindigkeit, wie auf die Klarheit resp. Trübung, so auch auf den Bakteriengehalt eines Flußwassers ausüben. Gegen das Vorjahr war die Zahl der Bakterien durchweg, und speziell in den Wintermonaten, erheblich vermehrt. Die starke Verunreinigung der Spree an der jetzigen Entnahmestelle der Stralauer Werke, welche u. a. auch in den hohen Zahlen der Mikroorganismen sich widerspiegelt, läßt eine Verlegung der Entnahmestelle nach einer vor Verunreinigungen besser geschützten Stelle der Oberspree, namentlich im Hinblick auf die Möglichkeit eines zeitweisen Versagens der Filteranlagen, z. B. am Ende eines harten, langdauernden Winters oder im Hochsommer bei plötzlicher starker Zunahme des Verlaufs, als ein dringendes Bedürfnis erscheinen. - Das Wasser des T e g e le r S e e s zeichnete sich, namentlich von Mitte September bis Mitte März, durch einen geringen Keimgehalt aus. Das verhältnismäßig ruhige Becken des Sees gibt offenbar den im Wasser suspendierten Bestandteilen, zu denen ja auch die Mikroorganismen und deren Keime gehören, hinreichende Gelegenheit zu Boden zu sinken und bildet so gleichsam ein großes Klärbassin. Nur vorübergehend, und zwar Anfang Juli und Ende März, machte sich eine starke Zunahme der Keime bemerkbar, welche vermutlich im ersteren Falle auf die für eine Vermehrung der Bakterien besonders günstige Jahreszeit, im letzteren Falle auf den starken Eisgang des Tegeler Sees zurückzuführen sein dürfte, dessen bereits im chemischen Teile dieses Berichtes Erwähnung geschah.

2. Beschaffenheit des Wassers nach der Filtration. Leistungender Filterwerke.

Ein Vergleich des Keimgehaltes des Wassers vor und nach der Filtration (Spalte 1 und 2 Stralau, Spalte 3 und 4 Tegel) läßt erkennen, daß die Beschaffenheit des durch Filtration gereinigten Wassers durchweg eine gute war, sowohl bei den Stralauer als bei den Tegeler Werken. Die starken Schwankungen im Bakteriengehalt des unfiltrierten Wassers, namentlich des Spreewassers, entsprechen keineswegs ähnlichen Schwankungen in der Beschaffenheit des Filtrats und der oft sehr beträchtliche Unterschied, welcher zwischen dem unfiltrierten Stralauer und Tegeler Wasser besteht, ist nach der Filtration, wie ein Vergleich der Spalten 2 und 4 ergibt, nicht mehr zu konstatieren. Es muß demnach anerkannt werden, daß beide Filterwerke während des Berichtzeitraumes in normaler Weise funktioniert haben. S t ö $\mathrm{r}$ u $\mathrm{n}$ g e $\mathrm{n}$ in der Wirksamkeit der Filter haben sich unter 88 Untersuchungen im ganzen 5mal, und zwar in Stralau an 3, in Tegel an 2 Untersuchungstagen durch plötzliches Auftreten zahlreicher Mikroorganismen in dem filtrierten Wasser bemerkbar gemacht. Nach Angabe der Herren Betriebs-Ingenieure P i e f k e und A n klamm war ein Grund für diese auffallenden übrigens schnell vorübergehenden Befunde in Gestalt einer unvermeidlichen Betriebsstörung in jedem einzelnen Falle nachzuweisen.

In Stralau stieg am 21. Juli der Keimgehalt des gereinigten Wassers von 200 auf 1656, und 2 Monate später, am 8. September, ebenso von 184 auf 1000 pro $\mathrm{ccm}$. Indes schon am nächstfolgenden Untersuchungstage, am 24. Juli resp. 15. September, war die Zahl der Organismen wieder auf die normale Höhe von 54 bzw. 44 zurückgegangen. Ein drittes Mal wiederholte sich derselbe Vorgang am 30. März. Zweimal in dem zuletzt 
erwähnten und im ersten Falle traf die mangelhafte Beschaffenheit des Filters mit einer besonders starken Verunreinigung des Spreewassers (100000 und mehr Keime pro ccm) zusammen, einmal, am 8. September, im Gegenteil mit einer auffallend geringen Verunreinigung, so daß an diesem Tage das Wasser nach der Filtration mehr Keime enthielt (1000) als unfiltriertes Spreewasser (960). Zu diesen Befunden gibt Herr Pie f k e nachfolgende Erläuterung:

„Das plötzliche und schnell vorübergehende Auftauchen zahlreicher Mikroorganismen im Leitungswasser erklärt sich aus dem Umstande, daß kurz vor den durch diese Erscheinung gekennzeichneten Tagen (21. Juli und 8. September) ein mit frischer Sandfüllung versehenes Filter in Betrieb genommen worden war. Die bakteriologișchen Beobachtungen, welche inzwischen auf dem Werke selbst angestellt worden sind, haben dargetan, daß frisch gewaschener Sand erst nach längerer Zeit (10-14 Tagen) genügend keimfreies Wasser liefert und daß es also notwendig ist, auf die Verwertung des ersten Filtrates zu verzichten. Die ungeheuren Ansprüche, denen das Filterwerk im Jahre $1885 \mathrm{zu}$ genügen hatte, machten jedoch eine solche Vorsichtsmaßregel unmöglich, auch fehlte es vor der fortlaufenden Anwendung der bakteriologischen Untersuchungsmethode im Betriebe selbst an klarer Einsicht über diesen Punkt. Nachdem dieselbe aber gewonnen, wird gerade die Spülung der Filter - nach jeder kürzeren oder längeren Betriebspause - mit besonderer Sorgfalt gehandhabt.

Der hohe Befund am 30. März 1886 ging aus der Bedrängnis hervor, in welche das Filterwerk durch den endlosen und harten Winter versetzt worden war. Die offenen Filter hatten sich sämtlich totgearbeitet, blieben aber wegen einer Eisdecke von 0,45 m Dicke unzugänglich und konnten nicht gereinigt werden. Die aktive Filterfläche hatte sich unter diesen Umständen bis auf weniger als $9000 \mathrm{qm}$ vermindert und das leider zu einer Zeit, wo das Wasser der Spree eine recht ungünstige Beschaffenheit hatte und eine besonders sorgfältige Filtration notwendig gewesen wäre."

In T e g e l wurden am 15. Dézember, nachdem das unfiltrierte Wasser daselbst seit Monaten nur einige hundert, speziell an diesem Tage 1290 Organismen im Kubikzentimeter enthalten hatte, $\mathrm{n}$ a $\mathrm{c} \mathrm{h}$ der Filtration 1500 Keime gefunden. 8 resp. 14 Tage früher betrug die Zahl 210 resp. 10, 8 resp. 14 Tage später 260 resp. 110. In geringerem Maße war dieselbe Erscheinung am 11. August beobachtet worden, an welchem Tage im filtrierten Tegeler Wasser 434 Organismen gegen 28 in der vorhergehenden und 50 in der folgenden Woche sich vorfanden. In beiden Filtern hatte kurz vor der Untersuchung eine Betriebsstörung stattgefunden, über welche Herr A n k la m m in nachstehender Weise berichtet:

„Am 10. August haben wir 2 Filter der neuen Anlage, welche periodisch bis zum 2. August im Betriebe waren, dann aber abgesperrt sind, mit der alten Anlage verbunden. Die Pumpe, an welcher am 11. August für die bakteriologische Untersuchung die Wasserprobe entnommen wurde, hat also teilweise Wasser enthalten, welches 8 Tage lang in den beiden Filtern und den zugehörigen Leitungen gestanden hat und in dem also die Keime inzwischen zur Entwicklung und Vermehrung gekommen waren.

Am 15. Dezember früh haben wir die alte Anlage zum Zweck von Rohranschlüssen (auf der Strecke nach Charlottenburg) außer Betrieb gesetzt. Bei derartigen Störungen in der Rohrleitung werden sich naturgemäß durch die großen Schwankungen der Wassersäule in den Röhren und Pumpen immer 
ältere Ablagerungen loslösen und dann von Einfluß auf den bakteriologischen Befund sein."

Das jedesmalige schnelle Verschwinden der vorgekommenen Unregelmäßigkeiten dürfte die Anschauung der Betriebsingenieure, wonach dieselben lediglich in den erwähnten, unvermeidlichen Betriebsstörungen und nicht etwa in mangelhafter Anlage oder fehlerhaftem Betriebe der Filter ihren Grund gehabt haben, durchaus bestätigen.

3. Der Einfluß des Hochreservoirs in Charlottenburg.

Es wurde bereits im ersten Teile dieses Berichtes bei Besprechung der physikalischen Eigenschaften erwähnt, daß das Hochreservoir in Charlottenburg zeitweise einen ungünstigen Einfluß auf die äußere Beschaffenheit des Wassers ausgeübt hat. Ein gleiches muß hinsichtlich des Bakteriengehaltes konstatiert werden, wie aus Spalte 5 der tabellarischen Übersicht und speziell aus einem Vergleich derselben mit Spalte 4 (filtriertes Wasser von Tegel) hervorgeht. Es zeigt das Wasser des Hochreservoirs zu 5 verschiedenen Malen, zuerst vorübergehend am 28. Juli und 20. Oktober, ferner in der Zeit vom 15. bis-29. Dezember, sodann Ende Januar und endlich fast während des ganzen Monats März einen zum Teil sehr erheblich höheren Bakteriengehalt als die in Tegel am Wasserwerke selbst geschöpfte Probe filtrierten Wassers.

Da eine Verunreinigung unterwegs in den Leitungsröhren zwischen Tegel und Charlottenburg auszuschließen sein dürfte, so werden wir den Grund dieser Erscheinung in den Verhältnissen der Charlottenburger Wasserstation selbst zu suchen haben. Dieselbe bildet bekanntlich einen Annex der Tegeler Werke, mit denen sie durch eine eiserne Druckröhrenleitung in Verbindung steht, und hat im wesentlichen die Aufgabe, einmal den Tegeler Pumpen einen Teil der Arbeit abzunehmen und außerdem den Tegeler Filtern als Reservoir zu dienen und denselben ein kontinuierliches, vollkommen gleichmäßiges Arbeiten dadurch zu ermöglichen, daß entsprechend den erheblichen Verschiedenheiten des Wasserbedarfs zu den verschiedenen Tagesstunden die Charlottenburger Pumpen stündlich wechselnde Wassermengen aus ihren Reservoirs in die Stadt drücken, während die Speisung des Reservoirs von Tegel aus ununterbrochen und gleichmäßig vor sich geht. Für die Stralauer Werke fehlt es bekanntlich an einer derartigen Reguliervorrichtung, da das vorhandene $2000 \mathrm{cbm}$ fassende Reinwasserreservoir bei einer Förderung von 40 bis $60000 \mathrm{cbm}$ täglich 20 bis $30 \mathrm{mal}$ seinen Inhalt nachholt, somit eigentlich nur als eine Saugekammer für die Pumpen dient. Die Arbeit der Filter kann daher im Leben keine kontinuierliche und gleichmäßige sein, wie in Tegel, wird sich vielmehr dem wechselnden Wasserverbrauch anpassen müssen. Die Station Charlottenburg besteht aus einem Pumpwerk und 3 großen gemauerten Reservoirs von $4 \mathrm{~m}$ Höhe und je 11 500, 11500 und 14000 , in Summa $37000 \mathrm{cbm}$ Raumgehalt. Da die tägliche Gesamtförderung sich auf $50-60000 \mathrm{cbm}$ beläuft, so wird der Inhalt des Reservoirs durchschnittlich täglich 2 mal erneuert. Infolge des unregelmäßigen Verbrauchs bei regelmäßigem Zufluß findet ein beständiges Schwanken des Wasserspiegels statt. Die Pumpen arbeiten nach einem vorher festgestellten, für längere Zeit in Geltung bleibenden Plan, welcher das stündlich zu fördernde Wasserquantum bestimmt. Nach Mitteilung des Betriebsingenieurs, Herrn Schaefer, würden sich unter Zugrundelegung einer Gesamtleistung von $50000 \mathrm{cbm}$ für die einzelnen Tagesstunden folgende Mengen ergeben, welche den Schwankungen im Wasserstande des Reservoirs umgekehrt entsprechen:

\begin{tabular}{|c|c|c|c|c|}
\hline & Uhr & lachts & 825 & $\mathrm{cbn}$ \\
\hline & , & , & 725 & \\
\hline & ", & ", & 725 & \\
\hline &, & ,. & 775 & \\
\hline
\end{tabular}




\begin{tabular}{|c|c|c|c|c|}
\hline \multicolumn{5}{|c|}{5 Uhr nachts $1100 \mathrm{cbm}$} \\
\hline 6 & $" 3$ & morgens & 1700 & 2 \\
\hline 7 & $"$ & , & 2425 & \\
\hline 8 & $"$ & $"$ & 2850 & \\
\hline 9 & , & , & 3050 & \\
\hline 10 & $"$ & , & 3050 & \\
\hline 11 & $"$ & , & 3000 & \\
\hline 12 & ," & נ, & 3000 & \\
\hline 1 &, 1 & mittags & 2725 & \\
\hline 2 & 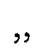 & , & 2725 & 3 \\
\hline 3 & $"$ & $"$ & 2725 & \\
\hline 4 & $"$ & $"$ & 2650 & \\
\hline 5 & $"$ & , & 2650 & , \\
\hline 6 & $"$ & , & 2500 & 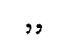 \\
\hline 7 & $"$ & 9 & 2275 & ", \\
\hline 8 & " & , & 2250 & \\
\hline 9 &, & , & 2075 & $"$ \\
\hline & ", & " & 1700 & \\
\hline & $"$ & ", & 1475 & \\
\hline & " & nachts & 900 & \\
\hline
\end{tabular}

Die Zirkulationsverhältnisse in den Reservoirs ergeben sich aus umstehender Skizze.

Das Wasser tritt aus dem Tegeler Hauptrohr in jedes Reservoir gesondert ein, und zwar in der einen Ecke, nahe dem Boden; der Austritt erfolgt in der Mitte der gegenüberliegenden Seitenwand, ebenfalls nahe dem Boden. Eine direkte Verbindung der Reservoirs untereinander besteht nicht; nur die zuführende und abführende Leitung sind gemeinsam. Letztere, aus 2 parallelen und untereinander in Verbindung stehenden Röhren bestehend, führt das Wasser aus allen 3 Reservoirs in eine gemeinsame gemauerte und überwölbte Saugekammer, aus welcher alsdann die Druckpumpen ihren Bedarf entnehmen. Der Zugang zu jedem Reservoir erfolgt durch eine an der einen Seitenwand gelegene gemauerte Treppe. Eine event. Verunreinigung des Wassers würde nur auf diesem, übrigens unter Verschluß gehaltenen Wege erfolgen können, da andere Zugänge fehlen.

Die Entnahme der zur Untersuchung bestimmten Wasserproben hat regelmäßig von einer dieser Treppen aus, und zwar meistens derjenigen des Reservoirs $I$ an der auf der Skizze mit a bezeichneten Stelle, stattgefunden.

In den obenerwähnten Fällen einer plötzlichen starken Zunahme der Bakterienmenge konnte nur durch die Untersuchung wiederholt konstatiert werden, daß die Hauptmasse derselben aus nur einer einzigen, übrigens ganz unschädlichen Bakterienart bestand, neben welcher sich die in dem Tegeler Wasser sonst gewöhnlich vorkommenden Arten in der üblichen geringen Anzahl von 50-150 vorfanden. Ohne Zweifel hatte also eine Bakterienvegetation stattgefunden. Die hierdurch bewirkte zeitweise Verunreinigung des Wassers kann indes nur von beschränktem Umfange gewesen sein und keineswegs die Gesamtmasse des Wassers betroffen haben, denn sie blieb, wie bereits im Eingange dieses Berichtes hinsichtlich der physilzalischen Eigenschaften hervorgehoben wurde und wie sich weiter unten auch für den Bakteriengehalt bestätigen wird, ohne Einfluß auf die Beschaffenheit des Leitungswassers in der Stadt. Eine Ausnahme macht die bereits erwähnte Störungsperiode Mitte Dezember, als infolge von Rohranschlüssen die Tegeler Werke mehrere Tage den Betrieb einschränken mußten (15.-17. Dezember). Infolgedessen war, wie oben bemerkt, schon in Tegel das filtrierte Wasser von mangelhafter 
Beschaffenheit ( 1500 Keime), und es scheint damals eine allgemeine Verunreinigung im Reservoir zu Charlottenburg stattgefunden zu haben (5000 Keime), welche sich auch auf die entsprechenden Entnahmestellen in der Stadt erstreckt hat und anscheinend sogar noch in der darauffolgenden Woche nachzuweisen war, wie weiter unten gezeigt werden soll. In den übrigen Fällen scheint es sich dagegen um eine vielleicht durch eine gewisse Stagnation in den Ecken der Reservoirs begünstigte lokale Verunreinigung in der Umgebung der Entnahmestelle gehandelt zu haben, welche auf die Hauptmasse des Wassers

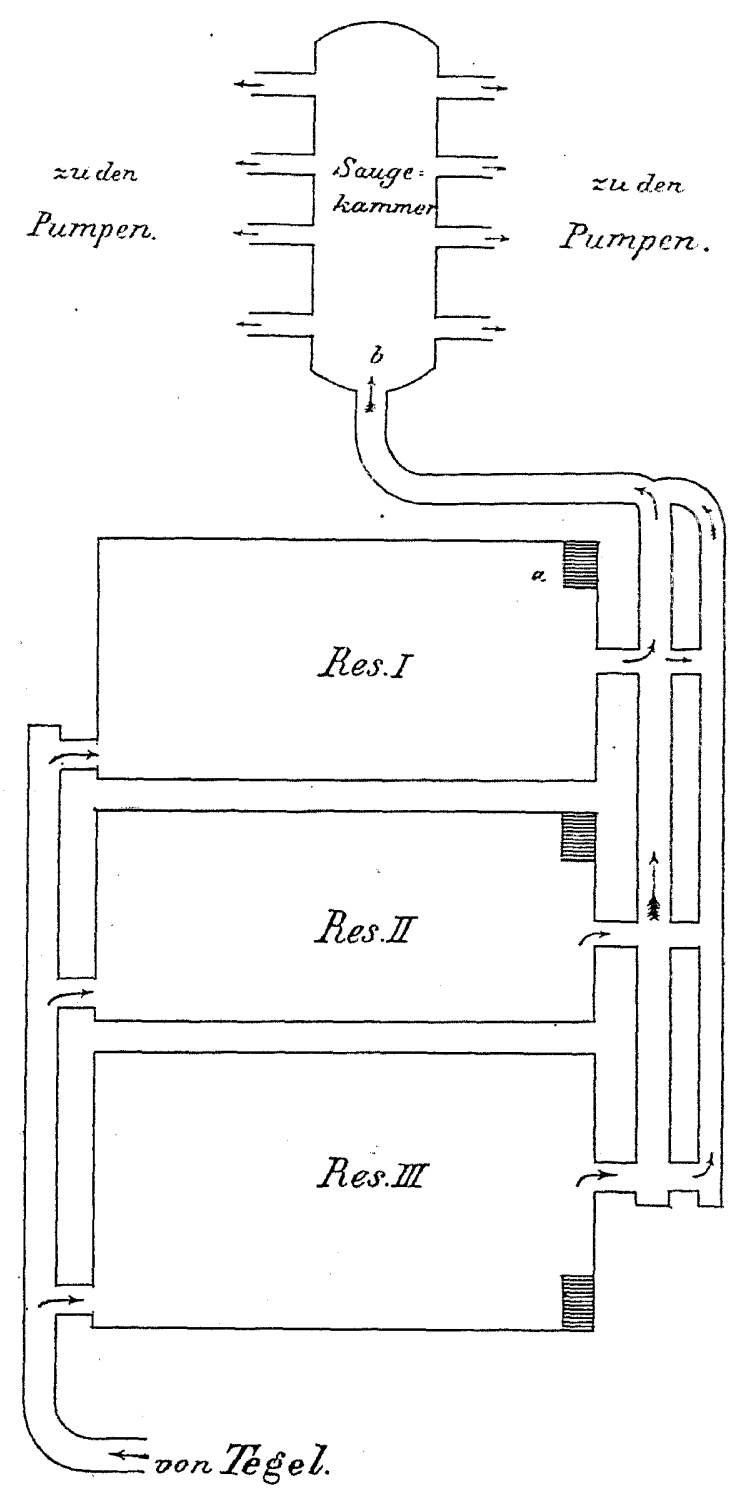

ohne Einfluß blieb. Um übrigens volle Sicherheit über diesen Punkt zu gewinnen, wird seit Anfang des laufenden Berichtsjahres die zur Untersuchung bestimmte Wasserprobe nicht mehr an der bisherigen Stelle, sondern durch eine Öffnung in der Decke der Saugekammer dicht vor der Einmündung des von den Reservoirs kommenden Zuleitungsrohres an der auf der Skizze mit b bezeichneten Stelle entnommen, wodurch die Gewinnung einer besseren Durchschnittsprobe gewährleistet erscheint. Zeitweise wurden auch vergleichende Untersuchungen der bisherigen und der neuen Entnahmestelle vorgenommen, 
über deren Ergebnis im nächsten Jahre berichtet werden soll. Noch bleibt zu bemerken, daß die zweimal im Jahre im Juli und November vorgenommene abwechselnde Reinigung der beiden damals allein vorhandenen Reservoirs I und II einen erkennbaren Einfluß auf die Beschaffenheit des Wassers nicht ausgeübt hat. Reservoir III ist neu erbaut und erst im laufenden Jahre in Betrieb genommen worden.

\section{Die Beschaffenheit des Wassers an den Entnahmestellen in der $\mathrm{Stad}$ t.}

Über die Beschaffenheit des Wassers in dem Rohrnetz der Stadt geben die Spalten 6, 7, 8, 9 und 10 der Tabelle Auskunft. Wenngleich die gefundenen Zahlen meist etwas höher sind als bei dem direkt den Reinwasserreservoirs zu Stralau und Tegel entnommenen Proben (Spalte 2 und 4), so ist das Resultat doch in der Hauptsache ein gleiches wie bei den Filterwerken, nämlich eine durchweg normale Beschaffenheit, mit einzelnen plötzlich auftretenden, aber schnell vorübergehenden Störungen. Der Grenzwert von höchstens 300 Mikroorganismen im Kubikzentimeter wurde unter je 44 Untersuchungen bei dem Wasser Nr. 62 mal,

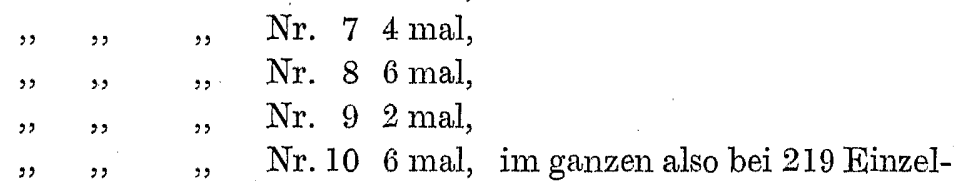
untersuchungen $20 \mathrm{mal}$ überschritten, während die übrigen 199 Untersuchungen ein zufriedenstellendes Resultat ergaben. Deutet dieser Befund schon darauf hin, daß das Wasser in den Leitungen eine erhebliche Veränderung nicht erfährt, vielmehr im wesentlichen in derjenigen Beschaffenheit zum Verbrauch gelangt, wie es von den Filterwerken geliefert wird, so läßt sich bei genauer Betrachtung auch für die einzelnen Entnahmestellen ein direktes Abhängigkeitsverhältnis von einem oder dem anderen Filterwerke nachweisen.

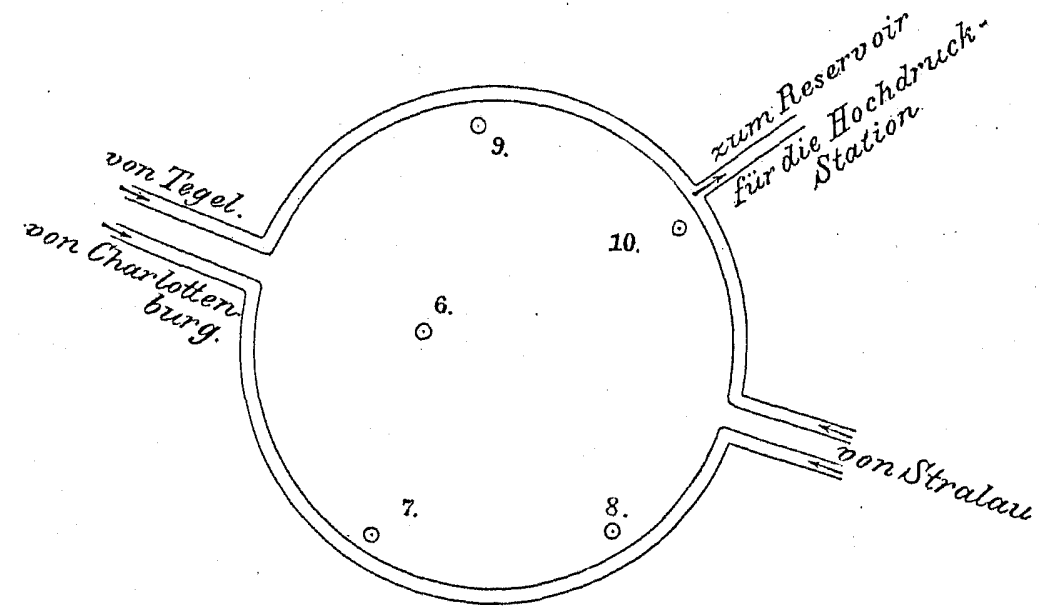

Bekanntlich gehen sowohl von Stralau als von Charlottenburg 2 Hauptdruckrohre aus, von denen je eins, der nördlichen resp. südlichen Peripherie der Stadt folgend, zuletzt mit dem entsprechenden Rohr des anderen Werkes zusammentrifft und mit demselben direkt kommuniziert. Auf diese Weise entsteht ein im allgemeinen an der Peripherie der Stadt gelegener geschlossener Ring, bestehend aus einem Rohr von 910 bis $760 \mathrm{~mm}$ Durchmesser, von dem aus durch Zwischenglieder und deren Verästelungen die Versorgung der ganzen Stadt erfolgt. Im Nordosten entspringt aus demselben noch das Hauptrohr für die Hochdruckstation, welche diesen höher gelegenen Stadtteil durch ein besonderes Rohrnetz mit Wasser versieht. cfr. die vorstehende Skizze. 
Die Lage der 5 Entnahmestellen ist nun eine derartige, daß 4 derselben, Nr. 7, 8, 9 und 10, in ziemlicher Nähe des Hauptrohres Nr. 6 dagegen in mehr zentraler Lage sich befinden. Dabei entspricht Nr. 7 dem südlichen, Nr. 9 dem nördlichen Druckrohr von Tegel, Nr. 8 dem südlichen, Nr. 10 dem nördlichen Druckrohr von Stralau.

Mit Hilfe der bereits obenerwähnten Differenzen im Chlorgehalt läßt sich nun leicht und sicher nachweisen, daß die Entnahmestellen 8 und 10, welche konstant den höheren Chlorgehalt des Spreewassers aufweisen, von Stralau aus, Nr. 6, 7 und 9 dagegen ron Tegel aus mit Wasser versorgt werden, Mittelzahlen aber, welche auf Mischwasser hindeuten würden, in der Regel fehlen.

Dies Verhältnis ist so konstant, daß der oben mehrfach erwähnte beschränkte Betrieb der Tegeler Anlage in der Zeit vom 15. bis 17. Dezember sich in der Tabelle des Chlorgehaltes sofort dadurch geltend machte, daß am 15. Dezember die Entnahmestellen 6, 7, 8 und 10 Spreewasser und nur 9 Tegeler Wasser, am 22. Dezember Nr. 6, 8 und 10 Spreewasser und 7 und 9 Tegeler Wasser und erst am 29. Dezember, ebenso wie vorher am 8. Dezember, Nr. 8 und 10 Spreewasser, Nr. 6, 7 und 9 Tegeler Wasser geliefert haben.

\begin{tabular}{|c|c|c|c|c|c|c|c|c|c|c|c|}
\hline \multirow{2}{*}{\multicolumn{2}{|c|}{ Tag der Untersuchung }} & \multirow{2}{*}{$\begin{array}{c}\text { Stralau } \\
\text { un- } \\
\text { filtriert } \\
1 . \\
\end{array}$} & \multirow{2}{*}{$\begin{array}{c}\text { Stralau } \\
\text { filtriert } \\
2 .\end{array}$} & \multirow{2}{*}{ 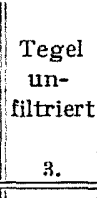 } & \multirow{2}{*}{$\begin{array}{c}\text { Tegel } \\
\text { filtriert } \\
4 . \\
\end{array}$} & \multirow{2}{*}{$\begin{array}{c}\text { Reser- } \\
\text { voir in } \\
\text { Char- } \\
\text { lotten- } \\
\text { burg } \\
5 .\end{array}$} & \multicolumn{5}{|c|}{ Entnahmestelle in der Stadt } \\
\hline & & & & & & & $\begin{array}{l}\text { W. } \\
6 . \\
\end{array}$ & $\begin{array}{c}S W . \\
\pi \\
\end{array}$ & $\begin{array}{c}\text { so. } \\
8 . \\
\end{array}$ & $\begin{array}{c}N . \\
\mathbf{9} . \\
\end{array}$ & $\begin{array}{r}\text { C. } \\
10 . \\
\end{array}$ \\
\hline 1385 Dezember & 8. & 24,9 & 24,9 & 16,0 & 16,0 & 16,0 & 16.0 & 16,0 & 24,9 & 16,0 & 24,9 \\
\hline Störuug in Tegel rom 15. & 15. & 24,9 & 24,9 & 16,0 & 16,0 & 16,0 & 24,9 & 24,9 & 24,9 & 16,0 & 24,9 \\
\hline bis 17. Dezember & 22. & 23,5 & 23,5 & 16,3 & 16,3 & 16,0 & 23,5 & 16,3 & 23,5 & 16,3 & 23,5 \\
\hline & 29. & 23,5 & 23,5 & 16,3 & 16.3 & 19,9 & 16,3 & 16,3 & 23,5 & 16,3 & 23,5 \\
\hline
\end{tabular}

Die auf Seite 422 befindliche Tabelle des Chlorgehalts gibt hierüber nähere Auskunft. In derselben sind die von der Regel abweichenden Befunde durch ein Kreuz bezeichnet ( + ).

Nach diesem Einblick in die Beziehungen der einzelnen Entnahmestellen zu den beiden Wasserwerken erscheinen auch die Ergebnisse der bakteriologischen Untersuchung in einem neuen Lichte. Es wird verständlich, weshalb die Spalten 8 und 10 der bakteriologischen Tabelle, in welchen je $6 \mathrm{mal}$ eine Überschreitung des Grenzwertes zu verzeichnen war, in dem Auftreten und Verschwinden dieser Störungen einen so auffallenclen Parallelismus zeigen, wie z. B. am 21. Juli, 24. November, 16. und 30. März. Weiterhin beweist aber die Gleichzeitigkeit dieser Störungen, daß die Ursache derselben auf dem Wasserwerke und nicht in den Leitungen liegen muß, weil beide Entnahmestellen zwar Stralauer Wasser, aber durch verschiedene Druckrohre erhalten (Nr. 8 durch das südliche, Nr. 10 durch das nördliche Hauptrohr von Stralau).

Für die von Tegel versorgten Entnahmestellen 6, 7 und 9 ist in bakteriologischer Hinsicht die Störungsperiode vom 15. bis 17. Dezember wieder von besonderem Interesse, da, übereinstimmend mit dem die Herkunft des Wassers kennzeichnenden Chlorgehalt, vom 15. Dezember an das Wasser Nr. 9 an der vorübergehenden Verunreinigung des Tegeler Wassers teilnimmt, während sich dieselbe in der folgenden Woche, wenn auch in abnehmendem Maße, auch bei Nr. 6 und 7 zu erkennen gibt.

Diese Umstände in Verbindung mit der regelmäßigen schnellen Wiederherstellung der normalen Beschaffenheit des Wassers dürften die oben aufgestellte Behauptung hinreichend erhärten, daß das Wasser in den Leitungen eine nennenswerte Veränderung nicht erfährt, seine Beschaffenheit vielmehr im wesentlichen von der jedesmaligen quanti- 
tativen Leistung der Wasserwerke abhängt. Hieraus folgt, daß der Schwerpunkt der Wasseruntersuchung in die Kontrolle der Filterwerke zu verlegen ist, die Untersuchung der einzelnen Entnahmestellen in der Stadt aber an Bedeutung verliert und diese eingeschränkt werden kann. Auf dieser Anschauung beruhten die Änderungsvorschläge, welche ich am 6. März d. J. ${ }^{1}$ ) dem Magistrat vorzulegen mich beehrte und welche seit Anfang Mai d. J., wenn auch in veränderter Form, durch Einrichtung einer bakteriologischen Untersuchungsstation auf dem Stralauer Wasserwerke unter gleichzeitiger Einschränkung der Untersuchungen im hygienischen Laboratorium auf eine 14tägige statt der bisherigen 8 tägigen $\mathrm{zu}$ einer teilweisen Ausführung gekommen sind.

$$
\text { 5. Das Vorkommen von Crenothrix. }
$$

Die Crenothrix scheint aus der Berliner Wasserleitung vollständig verschwunden zu sein. Sie wurde noch vereinzelt im Wasser der Charlottenburger Reservoirs und einzelner Entnahmestellen in der Stadt im Juni 1885, seitdem aber überhaupt nicht mehr beobachtet.

B erlin, am 12. November 1886.

$$
\begin{gathered}
\text { Der Direktor der hygienischen Institute. } \\
\text { Koch, } \\
\text { Geheimer Medizinalrat. }
\end{gathered}
$$

1) Diese Änderungsvorschläge enthalten schon die leitenden Gesichtspunkte, welche im Tahre 1899 (unter dem 13. Januar) unter Leitung des Kaiserl. Gesundheitsamtes als Grundsätze für die Reinigung von Oberflächenwasser durch Sandfiltration allgemeine Vorschrift geworden sind.

D. Herausgeber. 
Tabellarische Übersicht

über den Chlorgehalt im Berliner Leitungswasser.

\begin{tabular}{|c|c|c|c|c|c|c|c|c|c|c|c|}
\hline \multirow{3}{*}{\multicolumn{2}{|c|}{$\begin{array}{c}\text { Tag der } \\
\text { Lnter- } \\
\text { suchung }\end{array}$}} & \multirow{2}{*}{\multicolumn{2}{|c|}{$\frac{\text { Stralauer Werke }}{\text { Spreewasser }}$}} & \multicolumn{3}{|c|}{ Tegeler Werke } & \multicolumn{5}{|c|}{ Entuahmestellen in der Stadt } \\
\hline & & & & \multirow{2}{*}{$\begin{array}{c}\text { Wasser } \\
\text { unfiltriert } \\
3 .\end{array}$} & \multirow{2}{*}{$\begin{array}{c}\text { des Sees } \\
\text { filtriert } \\
44 .\end{array}$} & \multirow{2}{*}{$\begin{array}{c}\begin{array}{c}\text { Reservoir } \\
\text { in Char- } \\
\text { lotten- } \\
\text { burg }\end{array} \\
5 . \\
\end{array}$} & \multirow{2}{*}{$\begin{array}{c}\text { Wilhelm- } \\
\text { str. } 75 \\
W . \\
6 . \\
\end{array}$} & \multirow{2}{*}{$\begin{array}{l}\text { Friedrich- } \\
\text { str. } 41 / 42 \\
S W . \\
7 . \\
\end{array}$} & \multirow{2}{*}{ 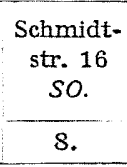 } & \multirow{2}{*}{$\begin{array}{c}\text { Friedrich- } \\
\text { str. } 126 \\
N . \\
9 . \\
\end{array}$} & \multirow{2}{*}{$\begin{array}{l}\text { Wein- } \\
\text { meister- } \\
\text { str. } 15 \\
\text { C. } \\
10 . \\
10 .\end{array}$} \\
\hline & & 1. & 2. & & & & & & & & \\
\hline \multicolumn{2}{|l|}{1885} & & & & & & & & & & \\
\hline \multirow[t]{5}{*}{ Juni } & 2. & 19,5 & 18,6 & 15.9 & $15,9^{\circ}$ & 15,9 & 15,1 & 15,1 & 17,7 & 15,9 & 18,6 \\
\hline & 9. & 19,5 & 18,6 & 15,9 & $15 ; 1$ & 15,9 & 15,9 & 15,1 & 18,6 & 15,9 & $19, \overline{3}$ \\
\hline & 16. & 19,5 & 18,6 & 17,7 & 15,9 & 15,9 & 15.9 & 16,8 & $17, i$ & 15,9 & 18.6 \\
\hline & 23. & 20,4 & 19,5 & 16,8 & 15,9 & 15,9 & 15,9 & 16,8 & 17,7 & 15.9 & 15,9 \\
\hline & 30. & 22,2 & 21,3 & 16,8 & 16,8 & 16,8 & 15,7 & 17.7 & 23,1 & 18,6 & 22,2 \\
\hline \multirow[t]{4}{*}{ Juli } & 7. & 21,3 & 18,6 & 16,8 & 15,9 & 15,9 & 16,8 & 15,9 & 19.5 & 15,9 & 17,7 \\
\hline & 14. & 21,3 & 21,3 & 15,9 & 15,9 & 15,9 & 15,9 & 16,8 & 18,6 & 15,9 & 18,6 \\
\hline & 21. & 21,3 & 20,4 & 15,9 & 15,9 & 15,9 & 15,1 & 15.1 & 20,4 & 15,9 & 19,5 \\
\hline & 28. & 22,2 & 20,4 & 15,9 & 15,9 & 15,9 & 16,8 & 15,9 & 20,4 & 15,9 & 21,3 \\
\hline \multirow[t]{4}{*}{ August } & 4. & 21,3 & 19,5 & 15,9 & 15,9 & 15,9 & 15,1 & 15,1 & 19,5 & 15,9 & 19,5 \\
\hline & 11. & 22,2 & 21,3 & 15,9 & 15,9 & 15,9 & 15,9 & 15,9 & 20,4 & 15,1 & 21,3 \\
\hline & 18. & 21,3 & 19,5 & 15,9 & 15,1 & 15,1 & 15,9 & 15,9 & 19,5 & 15,9 & 20,4 \\
\hline & 25. & 21,3 & 20,4 & 15,9 & 15.9 & 15,9 & 15,1 & 15.9 & 20,4 & 15,1 & $19, \overline{5}$ \\
\hline \multirow{2}{*}{\multicolumn{2}{|c|}{$\begin{array}{ll}\text { Seplember } 1 . & \\
& 8 .\end{array}$}} & 21,3 & 20,4 & 15,9 & 15,9 & 15,9 & 15.1 & 15,9 & 19,5 & 15,9 & 19,5 \\
\hline & & 21,3 & 21,3 & 16,8 & 15,1 & 1 & 15,9 & 15,9 & 21,3 & 15,9 & 20,4 \\
\hline & 15. & 22,2 & 21,3 & 15,9 & 15,1 & 15 & 15,1 & 15,9 & 20,4 & 15,9 & 21,3 \\
\hline & 22. & 31,9 & 25,7 & 28,1 & 21,3 & 21, & 19,5 & $26,6+$ & 28,9 & $24,8+$ & 28,9 \\
\hline & 29. & 30,8 & 30,8 & 16,3 & 16,3 & 16,3 & 14,5 & 16,3 & 30,8 & 16,3 & 26,8 \\
\hline \multirow[t]{4}{*}{ Oktober } & 6. & 30,8 & 30,8 & 16,3 & 16,3 & 16,3 & 16,3 & 16,3 & 30,8 & 16,3 & 29,8 \\
\hline & 13. & 30,8 & 30,8 & 16,3 & 16,3 & 16 & 16,3 & 16,3 & 27,2 & 16,3 & 30,8 \\
\hline & 20. & 32,0 & 32,0 & 16,0 & 16,0 & 16,0 & 16,0 & 16,0 & 32,0 & 16,0 & 32,0 \\
\hline & 27. & 30,2 & 30,2 & 16,0 & $16 ; 0$ & 16,0 & 16,0 & 16,0 & 30,2 & 16,0 & 19,5 \\
\hline \multirow{2}{*}{\multicolumn{2}{|c|}{$\begin{array}{r}\text { November } 3 . \\
10 .\end{array}$}} & 29,9 & 29,9 & 15,8 & 15,8 & 15,8 & 15,8 & 15,8 & 26.4 & 15,8 & 26,4 \\
\hline & & 26,6 & 26,6 & 16,0 & 16,0 & 16,0 & 14,2 & 14,2 & 21,3 & 14,2 & $21: 3$ \\
\hline & 17. & 26,6 & 26,6 & 16,0 & 16,0 & 16 & 16,0 & 16,0 & 26,6 & 16,0 & 26,6 \\
\hline & 24. & 24,9 & 24,9 & 16,0 & 16,0 & 16,0 & 16,0 & 16,0 & 24,9 & 16,0 & 24,9 \\
\hline Dezember & 1. & 28,1 & 23,1 & 16,0 & 16,0 & 16,0 & 16,0 & 16,0 & 23,1 & 16,0 & 23,1 \\
\hline & 8. & 24,9 & 24,9 & 16,0 & 16,0 & 16,0 & 16,0 & 16,0 & 24,9 & 16,0 & 24,9 \\
\hline & 15. & 24,9 & 24,9 & 16,0 & 16,0 & 16,0 & $24,9+$ & $24,9+$ & 24,9 & $16: 0$ & 24,9 \\
\hline & 22. & 23,5 & 23,5 & 16,3 & 16,3 & 16,3 & $23,5+$ & 16,3 & 23,5 & 16,8 & 23,5 \\
\hline & 29. & 23,5 & 23,5 & 16,3 & 16,3 & 19,9 & 16,3 & 16,3 & 28,5 & 16,3 & 23,5 \\
\hline 1886 & & & & & & & & & & & \\
\hline Januar & 5. & 22,6 & 22,6 & 16,3 & 16,3 & 18,1 & 16,3 & 18,1 & 22,6 & $22,6+$ & 19,9 \\
\hline & 12. & 23,5 & 23,5 & 16,3 & 16,3 & 16,3 & 16,3 & 16,3 & 21,7 & 16,3 & 23,5 \\
\hline & 19. & 23,5 & 28,5 & 18,1 & 19,9 & 18 & 18,1 & 18,1 & 23,5 & 18,1 & 23,5 \\
\hline & 26. & 21,7 & 21,7 & 16,0 & 17,8 & $17 ; 8$ & 16,0 & $21,7+$ & 21,7 & 17,8 & 21,7 \\
\hline Februar & 2. & 23,1 & 23,1 & 16,0 & 17,8 & 17,8 & 17,8 & 17,8 & 21,3 & 17,8 & 21,3 \\
\hline & 9. & 23,1 & 23,1 & 17,8 & 17,8 & 17,8 & 17,8 & 17,8 & 23,1 & 17,8 & 23,1 \\
\hline & 16. & 21,3 & 21,3 & 17,8 & 17,8 & 17,8 & 17,8 & 17,8 & 21,3 & 17,8 & 21,3 \\
\hline & 23. & 21,3 & 21,3 & 17,8 & 17,8 & 17,8 & 17,8 & 17,8 & 21,3 & 17,8 & 21,3 \\
\hline März & 2. & 22,3 & 22,3 & 18,3 & 18,3 & 16,5 & 16,5 & 16,5 & 22,3 & 16,5 & 22,3 \\
\hline & 9. & 20,1 & 20,1 & 16,5 & 16,5 & 16,5 & 16,5 & 16,5 & 20,1 & 16,5 & 18,3 \\
\hline & 16. & $20,1^{\circ}$ & 20,1 & 16,5 & 16,5 & 16,5 & 16,5 & 16,5 & 20,1 & 16,5 & 20,1 \\
\hline & 23. & 20,1 & 20,1 & 16,5 & 16,5 & 16,5 & 16,5 & 16,5 & 20,1 & 16,5 & 20,1 \\
\hline & 30. \| & 21,3 & 19,5 & 5,8 & 16,0 & 16,0 & 16,0 & 16,0 & 19,5 & 16,0 & 19,5 \\
\hline
\end{tabular}


Tabellarische Übersicht

über die im Wasser der Berliner Wasserleitung gefundenen Mikroorganismen.

NB. 1. Die Zahlen bezeichnen dieMenge der durch das Gelatinekulturverfahren in je1 ccm Wasser gefundenen Organismen. 2. Bei den filtrierten Wässern sind diejenigen Befunde, welche den zulässigen Grenzwert überschreiten, durch ein $(+)$ Krreuz, diejenigen, welche dens elben innehalten, durch einen $(-)$ Strich bereichnet. Als Grenzwert wurde die Anzahl von $300 \mathrm{~K}$ eimen pro ccm angenommen.

\begin{tabular}{|c|c|c|c|c|c|c|c|c|c|c|c|}
\hline & & Stralaue & r Werke & Teg & geler We & rke & & Entnahmes & stellen in & el Stadt & \\
\hline $\begin{array}{l}\text { Tag d } \\
\text { Unter } \\
\text { suchun }\end{array}$ & & $\begin{array}{r}\text { Spree } \\
\text { unfiltriert }\end{array}$ & $\begin{array}{l}\text { wasser } \\
\text { filtriert }\end{array}$ & $\begin{array}{l}\text { Wasser } \\
\text { unfiltriert }\end{array}$ & $\begin{array}{l}\text { des Sees } \\
\text { filtriert }\end{array}$ & $\begin{array}{c}\text { Reservoir } \\
\text { in Char- } \\
\text { lotten- } \\
\text { burg }\end{array}$ & \begin{tabular}{|} 
Wilhelm- \\
str. 75 \\
$W$.
\end{tabular} & $\begin{array}{c}\text { Friedrich- } \\
\text { str. } 41 / 42 \\
S W .\end{array}$ & $\begin{array}{c}\text { Schmidt- } \\
\text { str. } 16 \\
\text { SO. }\end{array}$ & $\begin{array}{c}\text { Friedrich- } \\
\text { str. } 126 \\
N:\end{array}$ & $\begin{array}{l}\text { Wein- } \\
\text { meister- } \\
\text { str. } 15 \\
C .\end{array}$ \\
\hline & & 1. & 2. & 3. & 4. & 5. & 6. & 7. & 8. & 9. & 10. \\
\hline 1885 & & & & & & & & & & & \\
\hline Juni & 2. & 5475 & $42-$ & 118 & $16-$ & $41-$ & $21-$ & $15-$ & $42-$ & $19-$ & $48-$ \\
\hline & 9. & 79 & $22-$ & 117 & $39-$ & $53-$ & $116-$ & $90-$ & $109-$ & $154-$ & $90-$ \\
\hline & 16. & 6100 & $33-$ & 115 & $76-$ & $41-$ & $130-$ & $66-$ & $42-$ & $132-$ & $115-$ \\
\hline & 23. & 6100 & $41-$ & 1325 & $194-$ & $104 \ldots$ & $125-$ & $684+$ & $50-$ & $145-$ & $84-$ \\
\hline & 30. & 4400 & $53-$ & 880 & $44-$ & $71-$ & $121-$ & $160-$ & $51-$ & $151-$ & $63-$ \\
\hline Juli & 7. & 3500 & $28-$ & verungluckt & $42-$ & $130-$ & $60-$ & $103-$ & $48-$ & $157-$ & $40-$ \\
\hline & 14. & 7200 & $200-$ & 1896 & $120-$ & $152-$ & $120-$ & $180-$ & $80-$ & $150-$ & $75-$ \\
\hline & 21. & 110740 & $1656+$ & 13220 & $49-$ & $90-$ & $136-$ & $240-$ & $368+$ & $107-$ & $810+$ \\
\hline & 28. & 2640 & $54-$ & 1500 & $48-$ & $31680+\mid$ & $151-$ & $156-$ & $63-$ & $85-$ & $86-$ \\
\hline August & 4. & 2310 & $70-$ & 900 & $28-$ & $209-$ & $95-$ & $141-$ & $53-$ & $96-$ & $75-$ \\
\hline & 11. & 00 & $65-$ & 1100 & $434+$ & $135-$ & $131-$ & $132-$ & $36-$ & $90-$ & $300-$ \\
\hline & 18. & 1800 & $36-$ & 179 & $50-$ & $70-$ & $300-$ & $149-$ & $125-$ & $113-$ & $140-$ \\
\hline & 25. & 11900 & $26-$ & 4410 & $21-$ & $40-$ & $180-$ & $164-$ & $37-$ & $96-$ & $59-$ \\
\hline Septembe & 1 & 3360 & $184-$ & 600 & $17-$ & $95-$ & $77-$ & $136-$ & $124-$ & $62-$ & $150-$ \\
\hline & 8. & 960 & $1000+$ & 1220 & $100-$ & $250-$ & $180-$ & $150-$ & $83-$ & verunglackt & $85-$ \\
\hline & 15. & 4500 & $44-$ & 158 & $56-$ & $106-$ & $154-$ & $139-$ & $67-$ & $275-$ & $134-$ \\
\hline & 22. & 9200 & $44-$ & 130 & $55-$ & $81-$ & $97-$ & $85-$ & $198-$ & $128-$ & $306+$ \\
\hline & 29. & 1120 & $30-$ & 111 & $31-$ & $82-$ & $76-$ & $98-$ & $63-$ & $74-$ & $87-$ \\
\hline Oktober & 6, & & - & 160 & $24-$ & $158-$ & $116-$ & & $104-$ & $5-$ & $29-$ \\
\hline & 13. & 1 & $25-$ & 519 & $29-$ & $90-$ & $125-$ & $67-$ & $62-$ & $75-$ & $32-$ \\
\hline & 20. & 2 & $36-$ & 174 & $18-$ & $720+$ & $132-$ & $78-$ & $18-$ & $28-$ & $41-$ \\
\hline & 27. & 4840 & $24-$ & 173 & $10-$ & $54-$ & $129-$ & $47-$ & $31-$ & $39-$ & $112-$ \\
\hline Norember & 3. & 8500 & $80-$ & 128 & $82-$ & $100-$ & $340+$ & $76-$ & $96-$ & $91-$ & $131-$ \\
\hline & 10. & 2. 520 & $42-$ & 250 & $32-$ & $120-$ & $284-$ & $38-$ & $126-$ & $34-$ & $62-$ \\
\hline & 17. & 6000 & $52-$ & 60 & $51-$ & $150-$ & $50-$ & $8-$ & $63-$ & $53-$ & $151-$ \\
\hline & 24. & 31500 & $167-$ & 251 & $78-$ & $66-$ & $90-$ & $29-$ & $580+$ & $18-$ & $540+$ \\
\hline Dezember & 1. & 9000 & $117-$ & 65 & $10-$ & $19-$ & $62-$ & $9-$ & $349+$ & $4-$ & $267-$ \\
\hline & 8. & & & 440 & $210-$ & $350+$ & $96-$ & $350+$ & & $244-$ & $300-$ \\
\hline & 15. & 5 & - & 1290 & $1500+$ & $5000+$ & $290-$ & $200-$ & $245-$ & $2880+$ & $155-$ \\
\hline & 22. & 5 & $34-$ & 86 & $260-$ & $350+$ & $320+$ & $390 \cdot+$ & $160-$ & $356+$ & $40-$ \\
\hline & 29. & 4000 & $20-$ & 149 & $110-$ & $950+$ & $196-$ & $300-$ & $56-$ & $131-$ & $56-$ \\
\hline 188 & & & & & & & & & & & \\
\hline Januar & 5. & 4500 & $95-$ & 80 & $38-$ & $205-$ & $81-$ & $75-$ & $74-$ & $45-$ & $40-$ \\
\hline & 12. & 1400 & $40-$ & 170 & $12-$ & $42-$ & $43-$ & $18-$ & $145-$ & $21-$ & $150-$ \\
\hline & 19. & 1 & $94-$ & 92 & $36-$ & $370+$ & $200-$ & $190-$ & $35-$ & $63-$ & $474+$ \\
\hline & 26 . & 29000 & $100-$ & 54 & $60-$ & $502+$ & $127-$ & $107-$ & $236-$ & $130-$ & $200-$ \\
\hline Februar & 2. & 20000 & $80-$ & 13600 & $24-$ & $98-$ & $173-$ & $107-$ & $260-$ & $70-$ & $250-$ \\
\hline & 9. & 5900 & $7-$ & 15 & $6-$ & $65-$ & $36-$ & $6-$ & $143-$ & $31-$ & $164-$ \\
\hline & 16. & & $10-$ & 30 & $2-$ & $75-$ & $26-$ & $22-$ & $80-$ & $103-$ & $50-$ \\
\hline & 23. & 1280 & $8-$ & 14: & $8-$ & $225-$ & $42-$ & $92-$ & $22-$ & $40-$ & $18-$ \\
\hline März & 2. & 1010 & $8-$ & 57 & $3-$ & $110-$ & $55-$ & $36-$ & $20-$ & $26-$ & $58-$ \\
\hline & 9. & 3680 & $112-$ & 225 & $19-$ & $475+$ & $165-$ & $163-$ & $105-$ & $266-$ & $42-$ \\
\hline & 16. & 14400 & $210-$ & 440 & $70-$ & $960+$ & $160-$ & $120-$ & $750+$ & $125-$ & $570+$ \\
\hline & 23. & 32700 & $145-$ & 16500 & $66-$ & $1870+$ & $136-$ & $130-$ & $130-$ & $50-$ & $200-$ \\
\hline & 30. & 100000 & $2300+$ & 50000 & $104-$ & $8800+1$ & $300-$ & $310+$ & $1600+$ & $200-1$ & $1100+$ \\
\hline
\end{tabular}

